\title{
Erratum: Steady-state cross-correlations for live two-colour super-resolution localization data sets
}

\author{
Matthew B. Stone \& Sarah L. Veatch
}

Nature Communications 6:7347 doi: 10.1038/ncomms8347 (2015); Published 12 Jun 2015; Updated 8 Sep 2015

This Article contains an error in Fig. 1 that was introduced during the production process. The inset in panel a should appear on top of the green and magenta dots, not beneath them. The correct version of the figure appears below.
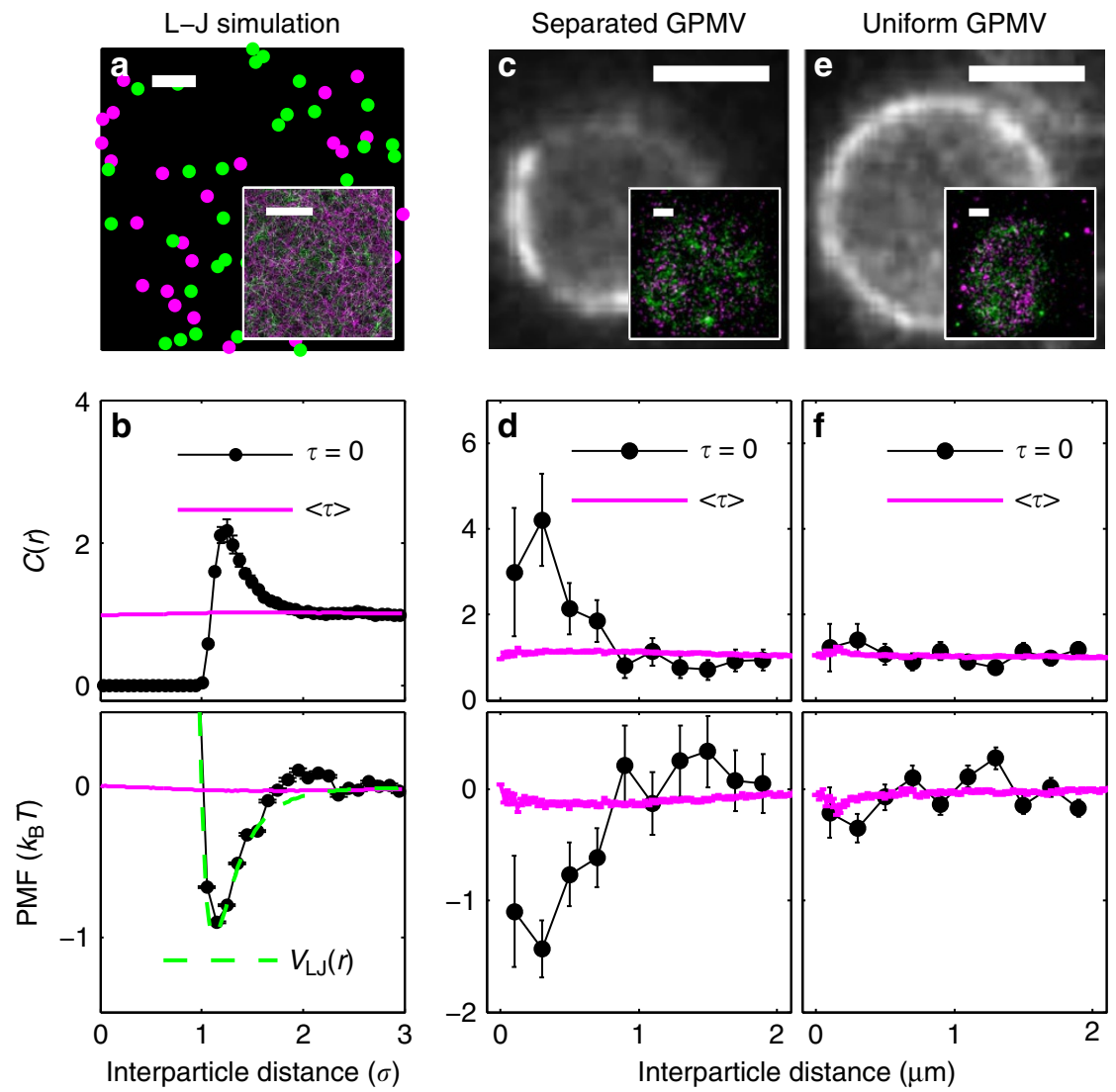

Figure 1 